\title{
SEPARATING POINTS BY SEMICHARACTERS IN TOPOLOGICAL SEMIGROUPS
}

\author{
JOHN W. BAKER AND NEAL J. ROTHMAN
}

1. The semigroup analogue of the Pontrjagin duality theorem was first studied in [1]. In that paper, it was shown that a necessary and sufficient condition for duality in discrete abelian semigroups is that the semigroup be a union of groups and have an identity element. Such semigroups we shall call inverse semigroups. For compact abelian topological semigroups it was shown in [1] that the separation of points by semicharacters is a sufficient condition for duality in an inverse semigroup with identity. In [2] it was shown that, in any topological abelian semigroup, a necessary condition for duality is that the semigroup be an inverse semigroup with identity and continuous inversion. In this paper we obtain necessary and sufficient conditions that semicharacters separate points in a topological abelian inverse semigroup with identity which is compact, or locally compact with continuous inversion. In the compact case we obtain the same result as has been given by Šneperman [5], using different methods.

1.1. Definition. An abelian semigroup is a nonempty set $S$ together with a map $m:(x, y) \rightarrow x y$ on $S \times S$ to $S$, such that $x(y z)=(x y) z$ and $x y=y x$ for all $x, y$ and $z$ in $S$. If $S$ is a Hausdorff topological space and the mapping $m$ is continuous, $S$ is called a topological abelian semigroup.

1.2. Definition. A semicharacter $\chi$ of a topological abelian semigroup $S$ is a bounded, continuous, complex-valued function on $S$, not identically zero, satisfying $\chi(x y)=\chi(x) \chi(y)$ for all $x$ and $y$ in $S$. We denote the set of semicharacters of $S$ by $S^{\wedge}$.

We endow $S^{\wedge}$ with the compact open topology. The following facts are to be found in [1], [3] or [4].

1.3. If $S$ has an identity element, $S^{\wedge}$ becomes a topological abelian semigroup, when endowed with the operation of pointwise multiplication.

1.4. If $S$ has an identity element, and is discrete, $S^{\wedge}$ is a compact abelian semigroup $[1,3.1]$.

1.5. We call $S$ an abelian inverse semigroup if $S$ is an abelian semigroup which is a union of groups. If $S$ is a topological, abelian inverse semigroup with an identity element then $S^{\wedge}$ is of the same type. Further, if $S$ is compact then $S^{\wedge}$ is discrete $[1,6.1]$.

Received by the editors March 26, 1968. 
1.6. If $S$ is a compact (or discrete), abelian inverse semigroup then the operation of inversion is a continuous map from $S$ to $S[6,2.4]$. If $S$ is a topological, abelian inverse semigroup with continuous inversion we call $S$ an abelian continuous-inverse semigroup.

1.7. If $I$ is an ideal in a topological abelian semigroup, then each element of $I^{\wedge}$ has a unique extension into an element of $S^{\wedge}[\mathbf{3}, 3.3 .3]$.

2. Let $S$ be a topological, abelian inverse semigroup, which has an identity element. Let $E$ denote the set of idempotent elements of $S$. For $e$ and $f$ in $E$ we write $e \leqq f$ if and only if $e f=e$ : thus $e \leqq f$ if and only if $e \in S f$. The relation $\leqq$ defines a partial order on $E$. For $e$ in $E, H(e)$ will denote the maximal subgroup of $S$ which contains $e$.

2.1. Definition. An idempotent $e$ in $S$ is said to be generating if $J_{e}=\bigcup\{H(f): f e \neq e\}$ is an open and closed, prime ideal in $S$. We observe that if $S$ is a continuous-inverse semigroup then $J_{e}$ is an open prime ideal in $S$ for every $e$ in $E$. Let $E_{g}$ denote the set of all generating idempotents in $E$. If $S$ is compact then $E_{g}$ is nonempty since it contains the idempotent in the minimal ideal.

Let $e$ and $f$ be elements of $E$ with $f \leqq e$. Let $\Pi_{f}^{e}: H(e) \rightarrow H(f)$ be defined by $\Pi_{f}^{e}(x)=f x$. It is easily seen that $\Pi_{f}^{e}$ is a continuous homomorphism. Further, if $f_{1} \leqq e$ and $f_{2} \leqq e$ then $f_{1} f_{2} \leqq e$ and $\Pi_{f_{1}}^{e} \Pi_{f_{1} f_{2}}^{f_{1}}$ $=\Pi_{f_{2}}^{e} \Pi_{f_{1} f_{2}}^{f_{2}}$. In particular, if $e$ is in $E$ and $\left\{e_{\alpha}\right\}$ is a net in $E$ with $e_{\alpha} / e$ and for each $\alpha$ and $\beta$ there exists $\gamma, \gamma>\alpha$ and $\gamma>\beta$ such that $e_{\gamma} e_{\alpha}=e_{\alpha}$ and $e_{\gamma} e_{\beta}=e_{\beta}$, then $\left[H\left(e_{\alpha}\right), \Pi_{e_{\beta}}^{e_{\alpha}}, \alpha>\beta\right]$ is an inverse system of topological groups.

2.2. Definition. The set $E_{g}$ of generating idempotents is said to be dense from below in $E$ if for each $e$ in $E$ there exists a net $\left\{e_{\alpha}\right\}$ in $E_{g} \cap S e$ such that $e_{\alpha} \rightarrow e$.

2.3. Lemma. Let $S$ be a compact, abelian inverse semigroup, with an identity element. If $E_{g}$ is dense from below, then for each e in $E$ there is a net $\left\{e_{\alpha}\right\}$ in $E_{\theta} \cap S e$ such that $e_{\alpha} \rightarrow e$ and $\alpha>\beta$ implies that $e_{\beta} \leqq e_{\alpha}$. Hence $\left[H\left(e_{\alpha}\right), \Pi_{e_{\beta}}^{e_{\alpha}}, \alpha>\beta\right]$ is an inverse system of compact groups. Finally $H(e) \approx \operatorname{proj} \lim \left[H\left(e_{\alpha}\right), \Pi_{e_{\beta}}^{e_{\alpha}}, \alpha>\beta\right]$.

Proof. If $e$ is in $E_{g}$ the conclusions are trivial. Let $e$ belong to $E \backslash E_{g}$ and let $V$ be a neighbourhood of $e$. Since $E_{g}$ is dense from below, there exists $e_{V}$ in $E_{g} \cap S e \cap V$. Now $U\left\{H(f): f e_{V} \neq e_{V}\right\}$ is open and closed and does not contain $e$. It follows that there is an open neighbourhood $W$ of $e$ such that $W \subseteq V, e_{V} \notin W$ and $f \in W \cap E$ implies that $f e_{V}=e_{V}$. By letting $V$ vary over a basis of open neighbourhoods of $e$, it is clear that we can construct a net $\left\{e_{\alpha}\right\}$ in $E_{g} \cap S e$ with $e_{\alpha} \rightarrow e$ and $\alpha>\beta$ implying $e_{\beta} \leqq e_{\alpha}$. The collection $\left[H\left(e_{\alpha}\right), \Pi_{e_{\beta}}^{e_{\alpha}}, \alpha>\beta\right]$ is clearly an 
inverse system of compact groups. Hence, only the isomorphism of $H(e)$ with the inverse limit remains to be proved.

Let us define $\Pi: H(e) \rightarrow \operatorname{proj} \lim \left[H\left(e_{\alpha}\right), \Pi_{e_{\beta}}^{e_{\alpha}}, \alpha>\beta\right]=G(e)$, say, by $\Pi(x)=\left(x e_{\alpha}\right)$. Then $\Pi$ is a continuous homomorphism of $H(e)$ into $\Pi\left[H\left(e_{\alpha}\right)\right]$. If $x \neq y$ are points of $H(e)$ then $x e_{\alpha} \rightarrow x e=x$ and $y e_{\alpha} \rightarrow y e=y$, so that $x e_{\alpha} \neq y e_{\alpha}$ for some $\alpha$. Hence $\Pi(x) \neq \Pi(y)$. For $\left(x_{\alpha}\right)$ in $G(e)$, $x_{\alpha} e_{\beta}=x_{\beta}$, for $\beta<\alpha$; and $\left\{x_{\alpha}\right\}$ is a net in $S$, a compact space. If $x$ is a cluster point of $\left\{x_{\alpha}\right\}$ it is clear that $\Pi(x)=\left(x_{\alpha}\right)$. Hence $\Pi$ is one-one and onto. Since $H(e)$ is compact, $\Pi$ is a homeomorphism. Hence $\Pi$ is the required isomorphism.

2.4. Lemma. Let $S$ be a locally compact, abelian, continuous-inverse semigroup. If $E$ is totally disconnected then $E_{g}$ is dense from below.

Proof. Let $e$ belong to $E$, and choose a compact, open neighbourhood $V$ of $e$. Clearly $E$ is closed in $S$, so that $E \cap V$ is also compact. We have to show that there exists $f$ in $E \cap V$ such that $f \leqq e$ and $f$ is in $E_{g}$. Treating $E \cap V$ as a partially ordered set under $\leqq$ we can use the compactness of this set to show that there is a minimal element $f$ in $E \cap V$ such that $f \leqq e$. If $f$ were not in $E_{g}$ then

$$
J_{j}=\bigcup\left\{H\left(e^{\prime}\right): e^{\prime} \in E \text { and } \quad e^{\prime} f \neq f\right\}
$$

is an open, but not closed, prime ideal in $S$. Let $\left\{x_{\alpha}\right\}$ be a net in $J_{f}$ with $x_{\alpha} \rightarrow x_{0} \in J_{f}$. Then $x_{\alpha}^{-1} \rightarrow x_{0}^{-1}$ in $S$, so that $e_{\alpha}=x_{\alpha} x_{\alpha}^{-1} \rightarrow e_{0}$ $=x_{0} x_{0}^{-1}$. Now $e_{0} f=f$ so that $e_{\alpha} f \rightarrow f$, and since $f$ is in $V$ there exists $\alpha$ so that $e_{\alpha} f$ is in $V$. Now $e_{\alpha} f \leqq f \leqq e$ and $f$ minimal imply that $e_{\alpha} f=f$; a contradiction. Hence $f$ is in $E_{g}$, and $E_{g}$ is dense from below.

2.5. We precede the main theorems with some remarks. Let $S$ be an abelian continuous-inverse semigroup with an identity element. In order to show that $S^{\wedge}$ separates points of $S$ it is sufficient to show that (i) if $x \neq y$ are in $H(e)$ then $S^{\wedge}$ separates $x$ and $y$ and (ii) if $e \neq f$ are in $E$ then $S^{\wedge}$ separates $e$ and $f$. But, if $e$ and $f$ are in $E$, we can separate $e$ and $f$ if we can separate either $e$ and $e f$ or $f$ and $e f$. Hence, it is sufficient in case (ii) to separate each $e$ and $f$ in $E$ for which $e \leqq f$.

If $e$ is in $E_{g}$ and $\chi_{0}$ is a character of $H(e)$ we can construct $\chi$ in $S^{\wedge}$ as follows. We define $\chi(x)=\chi_{0}(x)$ if $x$ is in $H(e)$ and $\chi(x)=0$ if $x$ is in $J_{e}$. Then $\chi$ is a (continuous) semicharacter on the ideal $J_{e} \cup H(e)$ and has a unique extension to $S$. It follows that $S^{\wedge}$ separates points of $H(e)$.

2.6. Theorem. Let $S$ be a compact, abelian, inverse semigroup with an identity element. The semigroup $S^{\wedge}$ separates points of $S$ if and only if $E$ is totally disconnected; in that case, for each $e$ in $E$ there exists a net 
$\left\{e_{\alpha}\right\}$ in $E_{g} \cap S e$ such that $e_{\alpha} \rightarrow e, e_{\alpha} e_{\beta}=e_{\beta}$ for $\alpha>\beta$ and $H(e)$ $\approx \operatorname{proj} \lim \left[H\left(e_{\alpha}\right), \Pi_{e_{\beta}}^{e_{\alpha}}, \alpha>\beta\right]$.

Proof. Clearly, if $S^{\wedge}$ separates points then $E$ is totally disconnected. Conversely, suppose that $E$ is totally disconnected. Since $S$ is compact, $S$ is a continuous-inverse semigroup. It is clear from Lemmas 2.4 and 2.3 that $E_{g}$ is dense from below and that each $H(e)$ is isomorphic to the required inverse limit. Recalling the remarks at the end of 2.5 , it is clear that we are then able to separate $x$ and $y$ in $H(e)$. On the other hand, if $e$ and $f$ are in $E$, with $f \leqq e$, choose $\left\{e_{\alpha}\right\}$ in $E_{g} \cap S e$ with $e_{\alpha} \rightarrow e$. Then $e_{\alpha} f \rightarrow f \neq e$, so that we can find $\alpha$ for which $e_{\alpha} f \neq e_{\alpha}$. Hence $f$ is in $J_{e_{\alpha}}$. As in 2.5 , we can construct $\chi$ in $S^{\wedge}$ with $\chi(f)=0$ and $\chi\left(e_{\alpha}\right)=1$. Since $e_{\alpha} \leqq e, \chi(e)=1$; so we can separate $f$ and $e$ by an element of $S^{\wedge}$. It follows that $S^{\wedge}$ separates the points of $S$.

2.7. In view of our introductory remarks we see that the Pontrjagin duality theorem is valid for a compact abelian semigroup $S$ if and only if $S$ is an inverse semigroup with identity element and $E$ is totally disconnected.

2.8. We remark that the isomorphism between $H(e)$ and the inverse limit in Theorem 2.6 may fail to be true even for a locally compact inverse semigroup. Let $Z$ denote the group of integers and $2 Z$ the even integers. Let $A$ be the semigroup $\{1\} \cup\left\{2^{-1 / n}: n=1\right.$, $2, \cdots\}$ with $x y=\min (x, y)$. Let $T=Z \times A$ and $S$ the subsemigroup $Z \times(A \backslash\{1\}) \cup(2 Z \times\{1\})$. Then $E_{o}=\{0\} \times(A \backslash\{1\})$, but $H(1) \approx 2 Z$ whereas proj $\lim \left[H(e), \Pi_{e}^{f}\right]=Z$.

2.9. Theorem. Let $S$ be a locally compact, abelian, continuousinverse semigroup, with identity element. The semigroup $S^{\wedge}$ separates points of $S$ if and only if $E$ is totally disconnected.

Proof. Clearly, if $S^{\wedge}$ separates the points of $S$ then $E$ is totally disconnected. Conversely, suppose that $E$ is totally disconnected. Lemma 2.4 shows that $E_{g}$ is dense from below. If $x \neq y$ are in $H(e)$ choose $\left\{e_{\alpha}\right\}$ in $E_{g} \cap S e$ with $e_{\alpha} \rightarrow e$. We can find $\alpha$ so that $e_{\alpha} x \neq e_{\alpha} y$. From 2.5 we see that $S^{\wedge}$ separates $e_{\alpha} x$ and $e_{\alpha} y$. If $\chi\left(e_{\alpha} x\right) \neq \chi\left(e_{\alpha} y\right)$ then $\chi\left(e_{\alpha}\right)=1=\chi(e)$ so that $\chi(x) \neq \chi(y)$. On the other hand, if $e \neq f$ are in $E$, with $f \leqq e$, we can separate $e$ and $f$ by a semicharacter as in Theorem 2.6. Hence $S^{\wedge}$ separates points of $S$.

\section{REFERENCES}

1. C. W. Austin, Duality theorems for some commutative semigroups, Trans. Amer. Math. Soc. 109 (1963), 245-256.

2. Anne C. Baker and J. W. Baker, Duality of topological semigroups with involution, J. London Math. Soc. 44 (1969), 251-260. 
3. A. B. Paalman de Miranda, Topological semigroups, Math. Centrum, Amsterdam, 1964, 174 pp.

4. S. Schwarz, The theory of commutative Hausdorff bicompact semigroups, Czechoslovak Math. J. 6 (1956), 330-361.

5. L. B. Sneperman, Representation of certain topological semigroups, Dokl. Akad. Nauk SSSR 176 (1967), 538-540=Soviet Math. Dokl. 8 (1967), 1157-1159.

6. A. D. Wallace, The structure of topological semigroups, Bull. Amer. Math. Soc. 61 (1955), 95-112.

Australian National University aNd UNIVERSITY OF ILLINOIS 